\title{
Spin-transfer torque in spin filter tunnel junctions
}

\author{
Christian Ortiz Pauyac, ${ }^{1}$ Alan Kalitsov, ${ }^{2}$ Aurelien Manchon, ${ }^{1, *}$ and Mairbek Chshiev ${ }^{2, \dagger}$ \\ ${ }^{1}$ King Abdullah University of Science and Technology (KAUST), Physical Science and Engineering Division, \\ Thuwal 23955-6900, Saudi Arabia \\ ${ }^{2}$ SPINTEC, UMR CEA/CNRS/UJF-Grenoble 1/Grenoble-INP, INAC, Grenoble, F-38054, France \\ (Received 29 May 2014; revised manuscript received 26 November 2014; published 8 December 2014)
}

\begin{abstract}
Spin-transfer torque in a class of magnetic tunnel junctions with noncollinear magnetizations, referred to as spin filter tunnel junctions, is studied within the tight-binding model using the nonequilibrium Green's function technique within Keldysh formalism. These junctions consist of one ferromagnet (FM) adjacent to a magnetic insulator (MI) or two FM separated by a MI. We find that the presence of the magnetic insulator dramatically enhances the magnitude of the spin-torque components compared to conventional magnetic tunnel junctions. The fieldlike torque is driven by the spin-dependent reflection at the MI/FM interface, which results in a small reduction of its amplitude when an insulating spacer (S) is inserted to decouple MI and FM layers. Meanwhile, the dampinglike torque is dominated by the tunneling electrons that experience the lowest barrier height. We propose a device of the form $\mathrm{FM} /(\mathrm{S}) / \mathrm{MI} /(\mathrm{S}) / \mathrm{FM}$ that takes advantage of these characteristics and allows for tuning the spin-torque magnitudes over a wide range just by rotation of the magnetization of the insulating layer.
\end{abstract}

DOI: 10.1103/PhysRevB.90.235417

PACS number(s): 85.75.-d, 73.40.Gk, 72.25.-b, 72.10.-d

\section{INTRODUCTION}

The intensive search for innovative devices enabling the efficient electrical control of magnetization direction has led to the discovery of spin-transfer torque (STT) [1]. In magnetic spin valves, the flowing charge current is polarized by the first (reference) magnetic layer and exerts a torque on the second (free) magnetic layer [2]. STT has emerged as an efficient mechanism to induce magnetization reversal and excitations, resulting in immediate applications such as magnetic random access memories (MRAM) and spin-torque oscillators, respectively [3]. Conventionally, the torque is partitioned into a fieldlike component, $T_{\perp}$, along $\boldsymbol{m} \times \boldsymbol{p}$, and a Slonczewski (dampinglike) component, $T_{\|}$, along $\boldsymbol{m} \times(\boldsymbol{m} \times \boldsymbol{p})$ direction, where $\boldsymbol{m}(\boldsymbol{p})$ represents the free(reference-) layer magnetization unit vector. Initially studied in metallic spin valves [4], STT was soon extended to magnetic tunnel junctions (MTJs), where the spacer was replaced by nonmagnetic insulating barriers such as $\mathrm{Al}_{2} \mathrm{O}_{3}$ [5] or $\mathrm{MgO}$ [6]. The latter, in contact with $\mathrm{Fe}(100)$ electrodes, captured the attention due to its crystallographic configuration that allowed coherent wave-function symmetry filtering, giving rise to a large tunneling magnetoresistance (TMR), as demonstrated theoretically [7] and experimentally [8]. Meanwhile, STT in $\mathrm{MgO}$-based tunnel junctions became the most promising ingredient for high-density, nonvolatile MRAM devices due to the combination of large TMR and low critical switching current. Substantial studies in ballistic regime $[9,10]$ revealed, for low bias $(V)$, the following form of the spin-torque components,

$$
\begin{gathered}
T_{\|}=a_{1} V+a_{2} V^{2}, \\
T_{\perp}=b_{0}+b_{1} V+b_{2} V^{2},
\end{gathered}
$$

\footnotetext{
*aurelien.manchon@kaust.edu.sa

†mair.chshiev@cea.fr
}

where the extra linear term of $T_{\perp}, b_{1}$, only appears due to symmetry breaking [12] originated from band filling mismatch of the electrodes [13], asymmetric barriers [14,15], or interfacial disorders [16].

Alternative tunneling systems have been proposed as an extension of conventional MTJs such as ferroelectrics and magnetic insulators. Ferroelectric junctions display electroresistance due to the electrical control of charge accumulation at the interfaces [17], whereas magnetic insulators exhibit spin filtering effect, which in contrast to symmetry filtering, selectively filters majority carriers due to the spin-dependent evanescence of the wave function in the barrier [18]. Consequently, even when considering a normal metal (NM) reference layer, higher spin polarization is obtained as a result of charge current being exponentially dependent on the exchange splitting of the barrier [19]. Magnetic insulators have attracted also increasing interest in magnonics and spin-caloritronics devices due to its ultralow damping coefficient [20]. Several architectures of spin filter based tunnel junctions (SFTJs) have been proposed, such as single junctions [21], double junctions [22], partial junctions [23], and symmetric junctions of the form FM/MI/FM [24]. These structures rely on either ferromagnetic or ferrimagnetic insulating barriers. The former includes Eu chalcogenides materials such as EuS [25], EuSe [26], and EuO [27], which present low Curie temperature $\left(T_{c}\right)$, i.e., $\sim 69 \mathrm{~K}$ [27]. The latter covers spinel-based materials such as $\mathrm{CoFe}_{2} \mathrm{O}_{4}$ [28], $\mathrm{NiFe}_{2} \mathrm{O}_{4}$ [29], $\mathrm{NiMn}_{2} \mathrm{O}_{4}$ [30], $\mathrm{BiMnO}_{3}$ [31], and $\mathrm{CoCr}_{2} \mathrm{O}_{4}$ [32], which exhibit $T_{c}$ values above room temperature. Most of the work on these architectures have focused on understanding spin polarization and TMR, where the latter tends to be much larger than conventional MTJs. However, the spin-transfer torque has been mostly overlooked and little has been done from a theoretical perspective [33].

In this work, the nature of spin-transfer torque in spin filter tunnel junctions is studied considering single-electron tight-binding (TB) calculations in the framework of Keldysh formalism [34]. The approach considers semi-infinite leads separated by a finite magnetic insulating barrier. Combined 
numerical and analytical studies as a function of bias in partial spin filter tunnel junctions (p-SFTJ), NM/MI/FM, reveal a dramatic enhancement of the torque amplitudes compared to conventional MTJs. The fieldlike component is driven by the spin-dependent reflection at MI/FM interface, which yields a linear bias dependence in contrast to the quadratic profile encountered in conventional MTJs and results in a small reduction of its amplitude when an insulating spacer (S) is inserted to break the exchange interaction between the magnetic insulator and the ferromagnet. Meanwhile, the dampinglike component is dominated by the tunneling electrons that experience the lowest barrier height. Based on these, we provide a route towards combining into a single device the STT mechanisms of conventional MTJs and partial SFTJs. For this, we introduce a structure of the form $\mathrm{FM} /(\mathrm{S}) / \mathrm{MI} /(\mathrm{S}) / \mathrm{FM}$, combining ferromagnetic metallic electrodes separated by a magnetic insulator, referred to as symmetric spin filter tunnel junction (s-SFTJ). This system allows for tuning the torques amplitude by rotating only the magnetization of the insulating layer.

This paper is organized as follows. Section II describes the theoretical method used to model spin filter tunnel junctions and to calculate the spin-transfer torques. Numerical and analytical studies of partial and symmetric spin filter tunnel junctions without insulating spacers are given in Sec. III A and III B, respectively. In Sec. III C, we present our results when spacers are inserted. In all cases, our solutions are compared with a conventional MTJ of similar parameters. In the following, to simplify the notation, we will call MTJ a system of the form FM/I/FM, where I represents the nonmagnetic insulating barrier of $N_{I}$ monolayers thickness. Finally, conclusions are summarized in Sec. IV.

\section{METHODOLOGY}

The Hamiltonian considered in the present study is described by the single-orbital simple-cubic TB Hamiltonian in noncollinear configuration, defined as

$$
\hat{H}=\hat{H}_{L}+\hat{H}_{R}+\hat{H}_{B}+\hat{H}_{\text {int }} .
$$

The first three terms correspond to the isolated contribution,

$$
\hat{H}_{\Omega}=\sum_{\substack{i \\(i \in \Omega)}} \epsilon_{\Omega, i}^{0} \hat{\boldsymbol{c}}_{i}^{\dagger} \hat{\boldsymbol{c}}_{i}+\Delta_{\Omega} \sum_{\substack{i \\(i \in \Omega)}} \hat{\boldsymbol{c}}_{i}^{\dagger} \hat{\boldsymbol{\sigma}} \cdot \hat{\boldsymbol{m}}_{\Omega} \hat{\boldsymbol{c}}_{i}+\sum_{\substack{i \neq i^{\prime} \\\left(i, i^{\prime} \in \Omega\right)}} t \hat{\boldsymbol{c}}_{i}^{\dagger} \hat{\boldsymbol{c}}_{i^{\prime}},
$$

$\Omega$ represents the uncoupled left $(\mathrm{L})$, right $(\mathrm{R})$, or barrier (B) regions. The last term defines the coupling with the leads,

$$
\hat{H}_{\mathrm{int}}=t \hat{\boldsymbol{c}}_{a}^{\dagger} \hat{\boldsymbol{c}}_{\alpha}+t \hat{\boldsymbol{c}}_{b}^{\dagger} \hat{\boldsymbol{c}}_{\alpha^{\prime}}+\text { H.c. },
$$

where, in $\hat{\boldsymbol{c}}_{i}^{\dagger}=\left(c_{i}^{\dagger \uparrow}, c_{i}^{\dagger \downarrow}\right), c_{i}^{\sigma}\left(c_{i}^{\dagger \sigma}\right)$ annihilates (creates) an electron with spin $\sigma$ on site $i(\in \Omega), \sigma=\uparrow(+1), \downarrow(-1)$ represents the up or down spin projection, and $t$ is the spin-independent hopping parameter between sites restricted to nearest neighbors, assumed to be constant everywhere $(t=$ $-1 \mathrm{eV})$. In Eq. (4), $\epsilon_{\Omega, i}^{0}=\left(\epsilon_{\Omega, i}^{\downarrow}+\epsilon_{\Omega, i}^{\uparrow}\right) / 2$ represents the averaged on-site energy in region $\Omega$ and site $i . \Delta_{\Omega}=\left(\epsilon_{\Omega}^{\downarrow}-\epsilon_{\Omega}^{\uparrow}\right) / 2$ define the exchange splitting, driven by the magnetization vector, $\hat{\boldsymbol{m}}_{\Omega}$, which is rotated in $x z$ plane with angle $\theta_{\Omega} . \hat{\boldsymbol{\sigma}}$ is the (a)

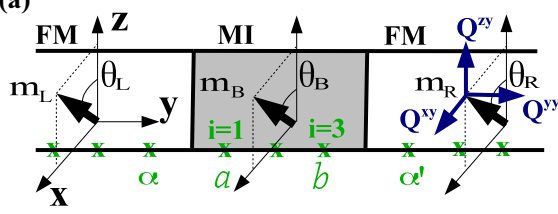

(c)
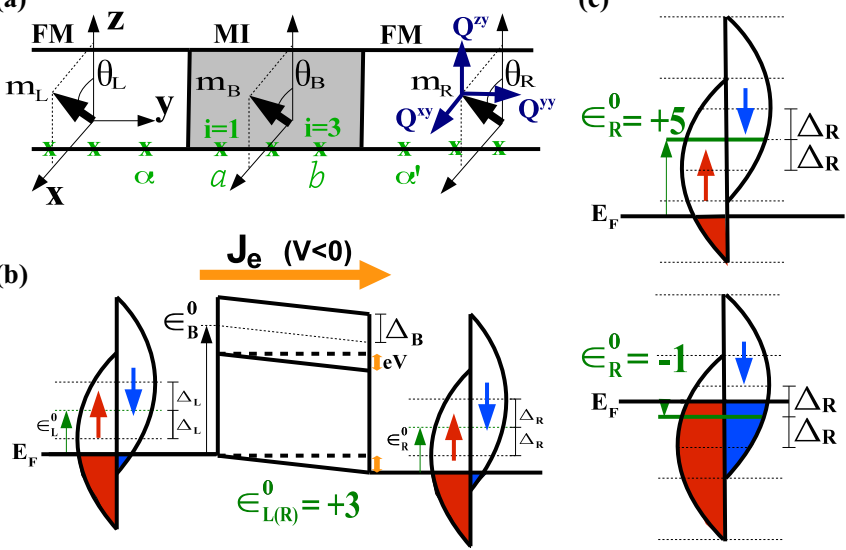

FIG. 1. (Color online) (a) Schematic structure of a SFTJ of the form FM/MI/FM. The magnetization vector on each layer is rotated by an angle $\theta$ in $x z$ plane. Subscripts L, R, and B stands for left, right, and barrier, respectively. The atomic layers, represented by green crosses, are enumerated from 1 to 3 in the barrier $\left(N_{B}=3\right)$. Subscripts next to left (right) interface are denoted $a(b)$ in the barrier and $\alpha\left(\alpha^{\prime}\right)$ in the lead. (b) Potential profile describing the averaged on-site energies $\left(\epsilon_{L}^{0}, \epsilon_{B}^{0}\right.$, and $\left.\epsilon_{R}^{0}\right)$ and exchange splittings $\left(\Delta_{L}, \Delta_{B}\right.$, and $\left.\Delta_{R}\right) . \mathrm{eV}=\mu_{R}-\mu_{L}$, with $\mu$ the chemical potential in the lead. $E_{F}=0 \mathrm{eV}$ refers to Fermi energy level. For negative bias, the electron flow density $\left(J_{e}\right)$ goes from reference layer (left) to free layer (right). (c) Density of states at zero bias for $\epsilon_{R}^{0}=+5$ and $-1 \mathrm{eV}$ cases.

Pauli matrix vector. Figure 1(a) shows the schematic structure of an ideal symmetric SFTJ-assumed periodic in $x z$ plane perpendicular to current direction along $y$-where insulating spacers between FM and MI are neglected. Subscripts $\alpha\left(\alpha^{\prime}\right)$ and $a(b)$ refer to atomic sites in left (right) and barrier regions, located next to FM/MI (MI/FM) interface. Figure 1(b) shows the potential profile where the averaged on-site energies and exchange splittings are described. Under applied bias the potential inside the barrier, given by $N_{B}$ monolayers, varies linearly with layer number $i, \epsilon_{B, i}^{\sigma}=\epsilon_{B}^{\sigma}+\operatorname{eV}(i-1) /\left(N_{B}-1\right)$. $\mathrm{eV}=\mu_{R}-\mu_{L}$, with $\mu$ the chemical potential in the lead. Insulating spacers of $N_{S}$ monolayers thick between FM and MI are modeled by modifying the interfacial barrier heights and taking $N_{B}=N_{\mathrm{MI}}+N_{S}$, where $N_{\mathrm{MI}}$ refers to the number of monolayers given just by the magnetic insulator. MTJ structures are modeled by considering $\Delta_{B}=0$ and taking $N_{B}=N_{I}$. A negative bias voltage corresponds to a negative charge current density; hence, the electron flow density, $J_{e}$, is defined positive when going from the reference layer (left) to the free layer (right). The isolated Green's function of each uncoupled region, $\Omega$, defined as $\hat{g}=\hat{I} /\left(E-\epsilon_{\boldsymbol{k}_{\|}}-\hat{H}_{\Omega}\right)$ is determined, where we have explicitly written $\epsilon_{\boldsymbol{k}_{\|}}$, being the energy of the in-plane vector, $\boldsymbol{k}_{\|}$, of the Bloch state. The system is then coupled through Dyson's equation [10]

$$
\hat{G}_{p q}=\hat{g}_{p q}+\hat{g}_{p a} \hat{\Sigma}_{a a} \hat{G}_{a q}+\hat{g}_{p b} \hat{\Sigma}_{b b} \hat{G}_{b q},
$$

where subscripts $p$ and $q$ are any two sites across the barrier, and the self-energy terms given by $\hat{\Sigma}_{a a(b b)}=t^{2} \hat{g}_{\alpha \alpha\left(\alpha^{\prime} \alpha^{\prime}\right)}$ take into account the propagation of the electron across barrier/electrode interfaces. Equation (6) is self-consistently solved and analytical expressions for the coupled retarded 
Green's functions, $\hat{G}_{p q}$, are obtained. To evaluate the net spin-transfer torque in the free layer, we proceed to solve the quantum kinetic equation based on Keldysh formalism [10], which yields solutions for the interfacial lesser Green's functions, $\hat{G}_{b \alpha^{\prime}\left(\alpha^{\prime} b\right)}^{<}$, of the form

$$
\hat{G}_{b \alpha^{\prime}\left(\alpha^{\prime} b\right)}^{<}=\hat{G}_{b \alpha^{\prime}\left(\alpha^{\prime} b\right)}^{<L}+\hat{G}_{b \alpha^{\prime}\left(\alpha^{\prime} b\right)}^{<R},
$$

with

$$
\begin{aligned}
& \hat{G}_{b \alpha^{\prime}}^{<L}=t^{2} \hat{D} \hat{B} \hat{g}_{b a}\left(\hat{I}+\hat{\Sigma}_{a a} \hat{A} \hat{g}_{a a}\right) \hat{g}_{\alpha \alpha}^{<} \hat{G}_{a \alpha^{\prime}}^{a}, \\
& \hat{G}_{b \alpha^{\prime}}^{<R}=t \hat{D} \hat{B}\left[\hat{g}_{b b}+\hat{g}_{b a} \hat{\Sigma}_{a a} \hat{A} \hat{g}_{a b}\right] \hat{g}_{\alpha^{\prime} \alpha^{\prime}}^{<}\left(\hat{I}+t \hat{G}_{b \alpha^{\prime}}^{a}\right),
\end{aligned}
$$

and

$$
\begin{aligned}
& \hat{G}_{\alpha^{\prime} b}^{<L}=t \hat{L} \hat{N} \hat{\Sigma}_{b b} \hat{g}_{b a} \hat{M} \hat{g}_{\alpha \alpha}^{<} \hat{G}_{a b}^{a}, \\
& \hat{G}_{\alpha^{\prime} b}^{<R}=t \hat{L} \hat{N} g_{\alpha^{\prime} \alpha^{\prime}}^{<} \hat{G}_{b b}^{a} .
\end{aligned}
$$

Superscript $a$ refers to the advanced Green's function, $\hat{g}_{\alpha \alpha\left(\alpha^{\prime} \alpha^{\prime}\right)}^{<}=-f_{L(R)}\left[\hat{g}_{\alpha \alpha\left(\alpha^{\prime} \alpha^{\prime}\right)}-\hat{g}_{\alpha \alpha\left(\alpha^{\prime} \alpha^{\prime}\right)}^{a}\right]$, with $f_{L(R)}$ the FermiDirac distribution in the left (right) layer. In these equations, $\hat{A}=\left(\hat{I}-\hat{g}_{a a} \hat{\Sigma}_{a a}\right)^{-1}, \hat{B}=\left(\hat{I}-\hat{g}_{b b} \hat{\Sigma}_{b b}\right)^{-1}$, and $\hat{D}=(\hat{I}-$ $\left.\hat{B} \hat{g}_{b a} \hat{\Sigma}_{a a} \hat{A} \hat{g}_{a b} \hat{\Sigma}_{b b}\right)^{-1}$; also, $\hat{N}=\left(\hat{I}-\hat{\Sigma}_{b b} \hat{g}_{b b}\right)^{-1}, \hat{M}=(\hat{I}-$ $\left.\hat{\Sigma}_{a a} \hat{g}_{a a}\right)^{-1}$, and $\hat{L}=\left(\hat{I}-\hat{N} \hat{\Sigma}_{b b} \hat{g}_{b a} \hat{M} \hat{\Sigma}_{a a} \hat{g}_{a b}\right)^{-1}$. The interfacial spin current density components $\left(Q_{b \alpha^{\prime}}^{j y}\right)$ and the electron flow density $\left(J_{e}\right)$ are obtained considering

$$
\begin{aligned}
Q_{b \alpha^{\prime}}^{j y} & =\frac{t}{4 \pi} \int \operatorname{Tr}\left[\left(\hat{G}_{\alpha^{\prime} b}^{<}-\hat{G}_{b \alpha^{\prime}}^{<}\right) \boldsymbol{\sigma}_{j}\right] d E d \boldsymbol{k}_{\|}, \\
J_{e} & =\frac{|e| t}{2 \pi \hbar} \int \operatorname{Tr}\left[\left(\hat{G}_{\alpha^{\prime} b}^{<}-\hat{G}_{b \alpha^{\prime}}^{<}\right)\right] d E d \boldsymbol{k}_{\|} .
\end{aligned}
$$

Superscript $j$ refers to the spin space direction, defined by the Pauli matrix, $\sigma_{j} . e$ and $t$ are the electric charge and hopping terms, respectively. $\hbar$ is the Planck's constant. $\boldsymbol{k}_{\|}$ is the transverse component of the wave vector, and the energy integral is over the whole energy range $[10,11]$. Finally, the Slonczewski and fieldlike components of the spin torque, $\mathbf{T}=\mathbf{Q}_{b \alpha^{\prime}}$, applied on the right lead are defined,

$$
\begin{gathered}
T_{\|} \equiv Q_{b \alpha^{\prime}}^{x y} \cos \theta_{R}-Q_{b \alpha^{\prime}}^{z y} \sin \theta_{R}, \\
T_{\perp} \equiv Q_{b \alpha^{\prime}}^{y y} .
\end{gathered}
$$

\section{RESULTS AND DISCUSSION}

In the simple cubic single-band tight-binding model, at zero bias, the energy limits of the density of states (DOS) are given by $E_{\min (\max )}^{\sigma}=\epsilon_{\Omega}^{\sigma} \pm 6 t$, which brings different band filling values in the leads associated with the DOS at Fermi level $\left(E_{F}=0 \mathrm{eV}\right)$. Four band filling cases are studied where $\Delta_{\Omega}=$ $+2 \mathrm{eV}$ unless stated otherwise; therefore, for the parameters considered here, $\epsilon_{L(R)}^{0}=+5 \mathrm{eV}$, given on Fig. 1(c) top panel, defines the half-metallic case, which considers no states at $E_{F}$ for minority carriers. $\epsilon_{R}^{0}=+3 \mathrm{eV}$, in contrast, allows a small contribution of minority spin states at Fermi level, see Fig. 1(b). Both cases are referred to be in the low-band-filling regime, which applies reasonably well to transition metals (Fe, $\mathrm{Co}, \mathrm{Ni}$ ) and their compounds. The tight-binding modeling of spin-transfer torque in $\mathrm{MgO}$-based tunnel junctions yields bias dependencies and magnitudes in semiquantitative agreement with the experimental observations [35]. We additionally investigate the cases $\epsilon_{R}^{0}=+1 \mathrm{eV}$ and $\epsilon_{R}^{0}=-1 \mathrm{eV}$, where the population of minority carriers at Fermi level increases until it becomes larger than the population of the majority carriers; therefore, a polarization inversion is expected as the system moves towards the high-band-filling regime, see Fig. 1(c) bottom panel. Our model being limited to a single band, it does not capture the specific wave-function symmetries that would arise in crystalline junctions, such as Fe/MgO [36]. However, limiting this study to low bias voltage, this model provides a qualitative description of the spin transport in spin filter junctions.

First, we investigate structures where insulating spacers needed to magnetically decouple the MI and FM layers are disregarded. Here, we study the bias dependence of STT in partial SFTJs (Sec. III A) and the angular dependence of STT in junctions of the form FM/MI/FM (Sec. III B). The former is set considering $\theta_{R(B)}=\pi / 2(0)$ and $\Delta_{L}=0$, the latter takes into account $\theta_{L(R)}=\pi / 2(0)$. In both cases, $\epsilon_{L}^{0}=\epsilon_{R}^{0}$ and the barrier height is set as close as possible to the Fermi level to guarantee large current densities. To avoid the Fowler-Nordheim effect [37], $\epsilon_{B}^{0}=+9 \mathrm{eV}$ and $\Delta_{B}=+2 \mathrm{eV}$ are considered, which brings at zero bias, $E_{\text {min }}^{\uparrow}=+1 \mathrm{eV}$. In Sec. IIIC, we study the case where insulating spacers are inserted between FM and MI in order to decouple the two magnetic layers. Such an insertion is in principle expected to dramatically reduce both TMR and STT amplitudes. However, it has been shown experimentally that insertion of a 0.8 -nm-thick $\mathrm{SrTiO}_{3}$ nonmagnetic insulating spacer between a FM electrode and a magnetic insulating barrier in $\mathrm{La}_{2 / 3} \mathrm{Sr}_{1 / 3} \mathrm{MnO}_{3} /\left(\mathrm{SrTiO}_{3}\right) / \mathrm{NiFe}_{2} \mathrm{O}_{4} / \mathrm{Au}$ junctions is efficient in decoupling the exchange interaction between the magnetic insulator and the electrode without substantially modifying the TMR ratio [29]. We show how the spintransfer torque components are modified when we insert the nonmagnetic insulating spacer. The dampinglike torque dramatically decreases in magnitude preserving its qualitative behavior as a function of bias. In contrast, the magnitude of the fieldlike torque is only slightly reduced in both partial and symmetric SFTJs. Therefore disregarding such spacers does not bring major changes in the fieldlike term, which should be measurable experimentally.

\section{A. Partial spin filter tunnel junction}

As displayed in Figs. 2(a) and 2(b), main panels, for the aforementioned band filling cases, it is found that a bilayer system of the form MI/FM is enough to dramatically enhance the spin-torque components compared to MTJs, displayed on insets. In Figs. 3(a) and 3(b), the torque efficiencies are displayed as a function of band filling for $V=-1.0 \mathrm{~V}$. Whereas the $T_{\|} / j_{e}$ magnitude remains on the same order as in MTJs, $\left(T_{\perp}-T_{\perp}^{0}\right) / j_{e}$ is increased by a factor of $10 \mathrm{in}$ the low-band-filling regions up to a factor of 100 as the system moves towards higher band filling values. Notice that the equilibrium interlayer exchange coupling (IEC) [38], i.e., the zero-bias value of $T_{\perp}, T_{\perp}^{0}$, has been subtracted. The underlying mechanism of the fieldlike torque is first understood in the framework of free-electron approach based on Wentzel-Kramers-Brillouin (WKB) approximation. In the 


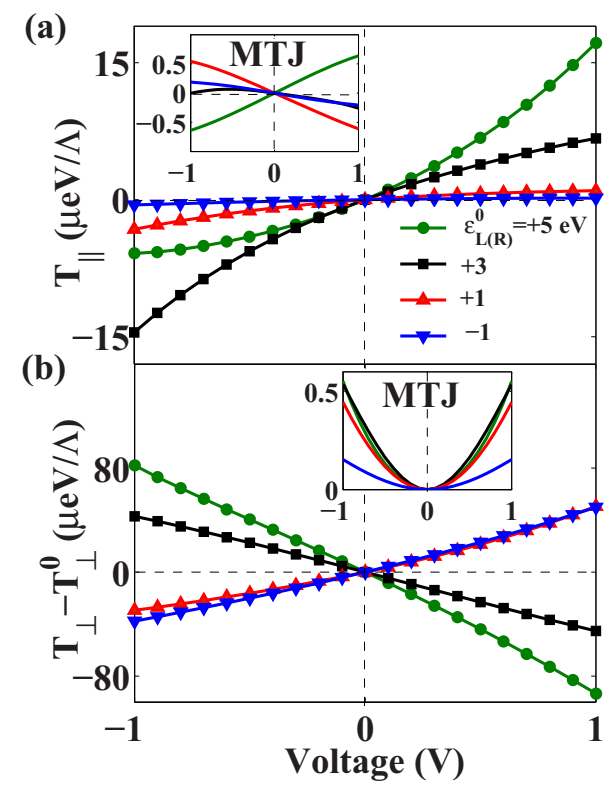

FIG. 2. (Color online) Bias dependence of nonequilibrium (a) Slonczewski, $T_{\|}$, and (b) fieldlike, $T_{\perp}-T_{\perp}^{0}$, spin-torque components, for $\mathrm{NM} / \mathrm{MI} / \mathrm{FM}$ (main panels) and FM/I/FM (insets) structures, with $\theta_{R}=\pi / 2, \theta_{L(B)}=0$ and $\epsilon_{B}^{0}=+9 \mathrm{eV}$ for different values of spin averaged on-site energies in the leads $\left(\epsilon_{L}^{0}=\epsilon_{R}^{0}\right) . \Delta_{L}=0$ and $\Delta_{R(B)}=+2 \mathrm{eV}$ in p-SFTJs (former). $\Delta_{B}=0$ and $\Delta_{L(R)}=+2 \mathrm{eV}$ in MTJs (latter). $N_{B}=N_{I}=N_{\mathrm{MI}}=3$ and the hopping parameter is $t=-1 \mathrm{eV}$. In all cases, the magnitudes of the torques are given in $\mu \mathrm{eV} / \Lambda$, where $\Lambda$ denotes the interfacial unit area.

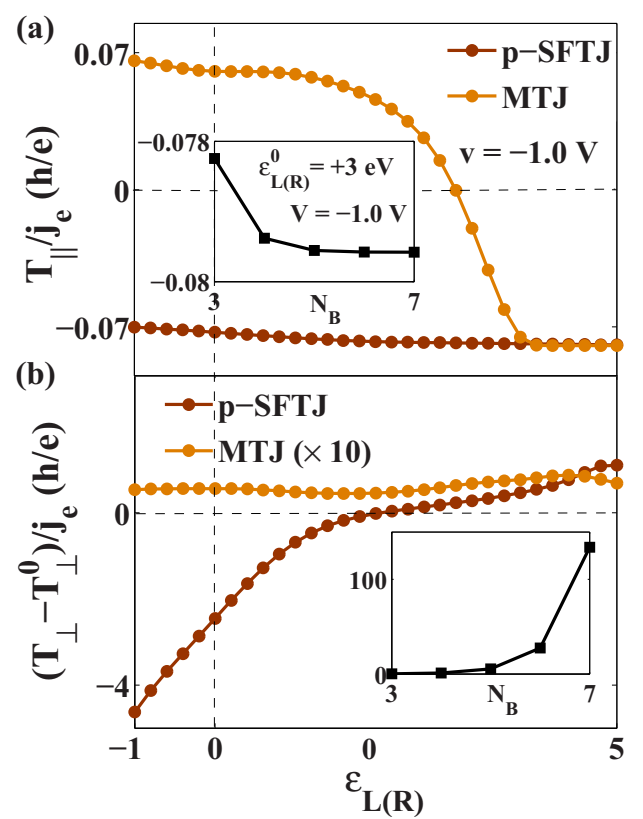

FIG. 3. (Color online) MTJ and p-SFTJ efficiencies for the nonequilibrium (a) Slonczewski and (b) fieldlike spin-torque components as a function of $\epsilon_{L(R)}^{0}$ and $V=-1.0 \mathrm{~V}$. Similar parameters as in Fig. 2 are considered. In (b), the MTJ magnitude is multiplied by 10 to fit in the graph. Insets refer to the torque efficiencies of the p-SFTJ as a function of barrier thickness $\left(N_{B}=N_{\mathrm{MI}}\right)$, given in numbers of atomic layers, for $V=-1.0 \mathrm{~V}$ and $\epsilon_{L(R)}^{0}=+3 \mathrm{eV}$. The magnitudes of the torque efficiencies are given in units of $h / e$. thick-barrier limit, to the lowest order in the transmission, the fieldlike component of the spin torque for electrons flowing from left to right $(L \rightarrow R)$ and right to left $(R \rightarrow L)$ read

$$
\begin{gathered}
T_{\perp}^{L \rightarrow R}=\frac{8 \hbar^{2}}{m \xi_{\theta_{R}}}\left(k_{R}^{\downarrow 2}-k_{R}^{\uparrow 2}\right) k_{L} q_{R}^{\downarrow} q_{R}^{\uparrow} \sum_{\sigma}\left(\sigma \frac{e^{-2 d \kappa^{\sigma}} q_{L}^{\sigma}}{k_{L}^{2}+q_{L}^{\sigma 2}}\right) \sin \theta_{R}, \\
T_{\perp}^{R \rightarrow L}=\frac{2 \hbar^{2}}{m \xi_{\theta_{R}}}\left(k_{R}^{\downarrow}-k_{R}^{\uparrow}\right)\left(q_{R}^{\downarrow}-q_{R}^{\uparrow}\right)\left(k_{R}^{\downarrow} k_{R}^{\uparrow}-q_{R}^{\downarrow} q_{R}^{\uparrow}\right) \sin \theta_{R} .
\end{gathered}
$$

$k_{R}^{\uparrow(\downarrow)}=\sqrt{\frac{2 m}{\hbar^{2}}\left(E \pm \Delta_{R}-\epsilon_{k_{\|}}+\mathrm{eV}\right)}, k_{L}=\sqrt{\frac{2 m}{\hbar^{2}}\left(E-\epsilon_{k_{\|}}\right)}$represent the wave vectors in the leads. In the barrier, the exponential decay is given by $e^{d \kappa^{\uparrow(\downarrow)}} \equiv e^{\int_{0}^{d} q_{B}^{\uparrow(\downarrow)} d y}$, with $q_{B}^{\uparrow(\downarrow)}=$ $\sqrt{2 m / \hbar^{2}\left(U_{o}-y \frac{\mathrm{eV}}{d} \mp \Delta_{B}-E+\epsilon_{k_{\|}}\right)} \cdot U_{o}$ and $d$ represent the barrier height and thickness, respectively. $m$ is the effective mass, $y$ is the transport direction and other parameters were already defined in the TB approach. For $y=0(d), q_{B}^{\uparrow(\downarrow)} \equiv$ $q_{L(R)}^{\uparrow(\downarrow)}$, and

$$
\begin{aligned}
\xi_{\theta_{R}}= & {\left[\left(k_{R}^{\downarrow}+k_{R}^{\uparrow}\right)\left(q_{R}^{\downarrow}+q_{R}^{\uparrow}\right)-\left(k_{R}^{\downarrow}-k_{R}^{\uparrow}\right)\left(q_{R}^{\downarrow}-q_{R}^{\uparrow}\right) \cos \theta_{R}\right]^{2} } \\
& +4\left(q_{R}^{\downarrow} q_{R}^{\uparrow}-k_{R}^{\downarrow} k_{R}^{\uparrow}\right)^{2} .
\end{aligned}
$$

In Eqs. (14) and (15), $\xi_{\theta_{R}}$ depends on the free layer magnetization direction and is driven by the exchange splitting of the barrier, see Eq. (16). This term brings a fieldlike torque angular dependence that deviates from the conventional $\sin \theta_{R}$ dependence encountered in MTJs. Within the TB formalism, this deviation, which appears also in $T_{\|}$, is small and becomes negligible in the high band filling regime. Of great importance is the nonvanishing zeroth-order term of $T_{\perp}^{R \rightarrow L}$, given in Eq. (15), which demonstrates that the fieldlike torque is dominated by the spin-dependent reflection at MI/FM interface, depending strongly on $q_{R}^{\uparrow}-q_{R}^{\downarrow}$ and $k_{R}^{\uparrow}-k_{R}^{\downarrow}$ and being independent of the barrier width. This result is confirmed in the TB approach. For this, in Eqs. (8) and (9), we vanish $G_{b \alpha^{\prime}\left(\alpha^{\prime} b\right)}^{<R}\left(G_{b \alpha^{\prime}\left(\alpha^{\prime} b\right)}^{<L}\right)$ to describe the interaction arising from left (right) layer only. It is found that even in the presence of a thin magnetic insulating barrier, for all band fillings, the nonequilibrium fieldlike torque, $T_{\perp}-T_{\perp}^{0}$, is driven by the spin-dependent reflection at MI/FM interface, and the contribution of the tunneling electrons $(L \rightarrow R)$ becomes negligible in high band filling regime. Meanwhile, the Slonczewski components, for electrons flowing from left to right and right to left, are both second order in barrier transmission, $\sim e^{-2 d \kappa^{\uparrow(\downarrow)}}$ (not shown here), and strongly depend on the barrier width.

On insets in Figs. 3(a) and 3(b), the torque efficiencies as a function of the barrier width for $\epsilon_{L(R)}^{0}=+3 \mathrm{eV}$ and $V=-1.0$ $V$ are displayed. Whereas $T_{\|} / j_{e}$ remains constant as a result of $T_{\|}$and $j_{e}$ decaying at the same rate, $\left(T_{\perp}-T_{\perp}^{0}\right) / j_{e}$ exponentially increases in agreement with Eq. (15). The torkances of the STT components, $\partial \boldsymbol{T} / \partial V$, as the system moves towards higher band filling, are of particular interest. First, in agreement with a recent work [33], due to the asymmetry of the junctions, the fieldlike torque depends linearly on the bias voltage; however, as $\epsilon_{L(R)}^{0}$ decreases, $\partial\left(T_{\perp}-T_{\perp}^{0}\right) / \partial V$ changes 
sign, see Fig. 2(b). The underlying mechanism is inferred from Eq. (15), $T_{\perp}^{R \rightarrow L} \propto\left(k_{R}^{\uparrow}-k_{R}^{\downarrow}\right)$, where the torkance changes sign as a result of polarization inversion. Meanwhile, the Slonczewski STT component exhibits an important feature in contrast to MTJs. Whereas in MTJs $\partial T_{\|} / \partial V$ changes sign as $\epsilon_{L(R)}^{0}$ decreases [see inset in Fig. 2(a)], in p-SFTJs it does not [see main panel in Fig. 2(a)]. This behavior is understood considering the WKB approximation, where $T_{\|}$for MTJs and p-SFTJs to the lowest order in the thick barrier limit are given by

$$
\begin{aligned}
T_{\|(\mathrm{MTJ})}^{L \rightarrow R} & =\frac{2 \hbar^{2}}{m} \frac{\left(k_{L}^{\downarrow}-k_{L}^{\uparrow}\right)\left(k_{R}^{\downarrow}+k_{R}^{\uparrow}\right)\left(k_{L}^{\downarrow} k_{L}^{\uparrow}-q_{L}^{2}\right)\left(k_{R}^{\downarrow} k_{R}^{\uparrow}+q_{R}^{2}\right) q_{L} q_{R} e^{-2 d \kappa}}{\left(k_{L}^{\downarrow 2}+q_{L}^{2}\right)\left(k_{L}^{\uparrow 2}+q_{L}^{2}\right)\left(k_{R}^{\downarrow 2}+q_{R}^{2}\right)\left(k_{R}^{\uparrow 2}+q_{R}^{2}\right)} \sin \theta_{R} \equiv \mathscr{T}_{L} \mathscr{P}_{L} \sin \theta_{R}, \\
T_{\|(\mathrm{pSFTJ})}^{L \rightarrow R} & =\frac{8 \hbar^{2}}{m \xi_{\theta_{R}}}\left(k_{R}^{\downarrow}+k_{R}^{\uparrow}\right) k_{L}\left[\frac{e^{-2 d k^{\uparrow}} q_{L}^{\uparrow} q_{R}^{\uparrow}\left(k_{R}^{\downarrow} k_{R}^{\uparrow}+q_{R}^{\downarrow 2}\right)}{k_{L}^{2}+q_{L}^{\uparrow 2}}-\frac{e^{-2 d k^{\downarrow}} q_{L}^{\downarrow} q_{R}^{\downarrow}\left(k_{R}^{\downarrow} k_{R}^{\uparrow}+q_{R}^{\uparrow 2}\right)}{k_{L}^{2}+q_{L}^{\downarrow 2}}\right] \sin \theta_{R} \\
& \approx \frac{8 \hbar^{2}}{m \xi_{\theta_{R}}}\left(k_{R}^{\downarrow}+k_{R}^{\uparrow}\right) k_{L}\left[\frac{e^{-2 d \kappa^{\uparrow}} q_{L}^{\uparrow} q_{R}^{\uparrow}\left(k_{R}^{\downarrow} k_{R}^{\uparrow}+q_{R}^{\downarrow 2}\right)}{k_{L}^{2}+q_{L}^{\uparrow 2}}\right] \sin \theta_{R} .
\end{aligned}
$$

For simplicity, we have considered the contribution of electrons incident from the left layer only. $\mathscr{T}_{L}$ and $\mathscr{P}_{L}$, defined in Ref. [38], are the transmissivity and effective Slonczewski polarization of left layer, respectively. It is straightforward to notice from Eq. (17) that in MTJs the sign of $T_{\|}$is determined by the sign of the polarization $\mathscr{P}_{L} \propto\left(k_{L}^{\downarrow}-k_{L}^{\uparrow}\right)$, whereas in p-SFTJs, given in Eq. (18), $\mathscr{P}_{L}$ is absent and no sign reversal is expected since $T_{\|}$is driven by the tunneling electrons that experience the lowest barrier height [majority electrons in Eq. (18)].

\section{B. Symmetric spin filter tunnel junction}

In the previous section, we have seen that a magnetic insulating barrier adjacent to a ferromagnetic layer dramatically enhances the torque amplitude compared to magnetic tunnel junctions. The fieldlike torque is driven by the spin-dependent reflection at the MI/FM interface, whereas the Slonczewski component is dominated by the tunneling electrons that experience the lowest barrier height. To exploit these important features, we propose a device of the form FM/MI/FM, referred to as symmetric spin filter tunnel junction, or s-SFTJ. The system now consists of two ferromagnets separated by a magnetic insulator, the left layer is referred to as the reference layer and the right layer is the free layer on which the torque is exerted. In this device, when the magnetization direction of the barrier is aligned on the magnetization of the free layer, the junction is expected to behave like an MTJ. In contrast, when the magnetization of the barrier is misaligned with respect to the magnetization of the free layer, the system behaves like the p-SFTJ discussed in the previous section. Therefore, the magnitude of the spin-transfer torques can be tuned, depending on the relative angle between the magnetizations of the barrier and free layer. In Figs. 4(a) and 4(b), we display the angular dependence of the STT components for $V=+1.0 \mathrm{~V}$. For this study, the magnetization of the barrier is rotated by an angle $\theta_{B}$ while the magnetization of the leads is kept perpendicular to each other, $\theta_{L(R)}=\pi / 2(0)$, as sketched in Fig. 4(b). When the magnetization of the barrier is perpendicular to the magnetization of the free layer, the fieldlike torque is driven by the spin-dependent reflection. Thus, as shown in Fig. 4(d) (bottom-right panel), where we have plotted the case $\epsilon_{L(R)}^{0}=+3 \mathrm{eV}$ and $\theta_{B}=3 \pi / 2$ for $\mathrm{s}-\mathrm{SFTJ}$ and p-SFTJ junctions, the fieldlike torque is likely to be independent on the details of the electronic structure of the reference layer and therefore displays a bias dependence similar to partial SFTJs. In contrast, when the barrier is aligned or antialigned to the free layer, Eqs. (14) and (15) vanish; therefore, the contribution to the fieldlike torque is driven by the leads, recovering MTJ bias characteristics, as shown in Fig. 4(d) (bottom-left panel) for the case $\theta_{B}=0$, where the slight enhancement is due to the spin-filtering imposed by the magnetic insulating barrier. Similar outputs are found in other band filling cases. Consequently, at low voltage, the bias dependence of the nonequilibrium field like torque in s-SFTJs can be expressed as

$$
T_{\perp}-T_{\perp}^{0}=b_{1\left(\theta_{B}\right)} V+b_{2\left(\theta_{B}\right)} V^{2},
$$

where the coefficients, in contrast to Eq. (2), depend on $\theta_{B}$. The Slonczewski STT component shows a more complex angular behavior, which depends also on band filling regime and on the relative orientation of the magnetizations in the barrier and left layer. For the half-metallic case, depicted by the filled green dots in Fig. 4, the reference layer filters the spin states. If these states are anti-aligned with the magnetization of the barrier $\left(\theta_{B}=3 \pi / 2\right)$, the electron flow density exponentially drops, as shown in Fig. 4(c); therefore, $T_{\|}$is dramatically reduced, see Fig. 4(a). However, if the spin states are aligned with the magnetization of the barrier $\left(\theta_{B}=\pi / 2\right)$, the enhanced electron tunneling results in a dramatic increase in the torque amplitude and in the electron flow density, see Figs. 4(a) and $4(\mathrm{c})$. Furthermore, when the magnetization of the barrier is aligned on the magnetization of the free layer $\left(\theta_{B}=0\right)$, the system behaves like a MTJ with an enhancement in the torque amplitude due to the magnetic nature of the insulating layer. This effect persists for higher band filling values, as displayed in Fig. 4(d), top-left panel, for the case $\epsilon_{L(R)}^{0}=+3 \mathrm{eV}$. An important feature in nonhalf metallic cases appears when the magnetization of the barrier is antiparallel $\left(\theta_{B}=3 \pi / 2\right)$ or parallel $\left(\theta_{B}=\pi / 2\right)$ to the magnetization of the reference layer. 

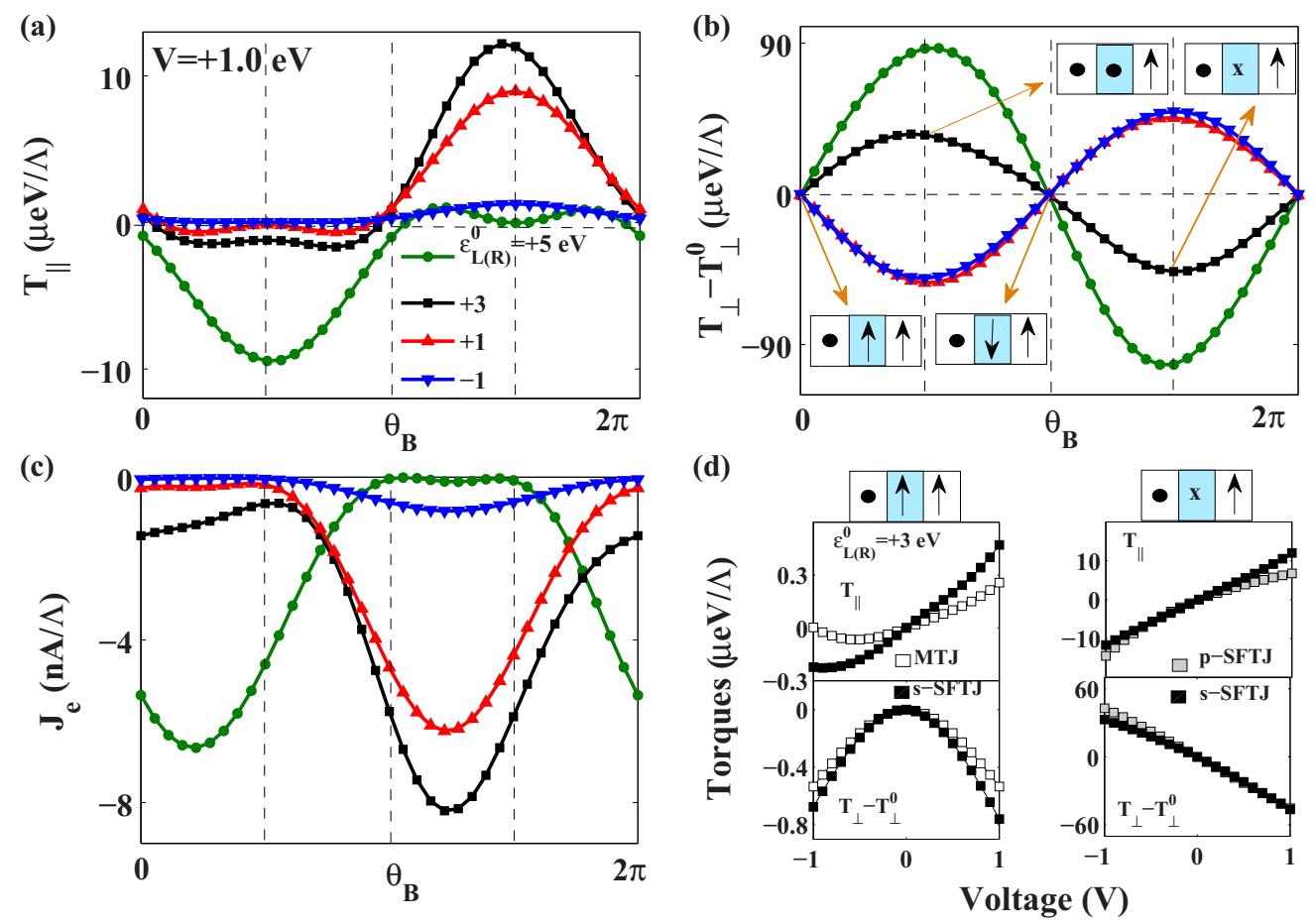

FIG. 4. (Color online) Angular dependence of nonequilibrium (a) Slonczewski torque, (b) fieldlike torque, and (c) electron flow density, for FM/MI/FM structure (s-SFTJ) with $V=+1.0 \mathrm{~V}$ and four values of the spin-averaged on-site energy. (d) Bias dependence of the spin-torque components for $1 / 4$ band filling case $\left(\epsilon_{L(R)}^{0}=+3 \mathrm{eV}\right)$. Left- (right-) hand insets compare the s-SFTJ solutions with a MTJ (p-SFTJ) structure with parameters similar to those given in Fig. 2. The magnetization of the barrier is rotated by an angle $\theta_{B}$ while the magnetization of the leads are kept perpendicular to each other, $\theta_{L(R)}=\pi / 2(0) . \Delta_{L(R)(B)}=+2 \mathrm{eV}$ in s-SFTJ.

In the former, given in Fig. 4(d) top-right panel, for $\epsilon_{L(R)}^{0}=$ $+3 \mathrm{eV}, T_{\|}$behaves like in partial SFTJs, whereas in the latter, the magnitude is reduced, see Fig. 4(a). To understand these effects, we recall the discussion in the previous section, where we mentioned that $T_{\|}$in p-SFTJs is driven by the tunneling electrons that experience the lowest barrier height, implying that when the polarization inversion is reached (high-bandfilling regime), $J_{A P}>J_{P}$, being $J_{A P(P)}$ the charge current density in antiparallel (parallel) configuration, $\theta_{B}=0$ and $\theta_{R}=\pi(0)$. Similarly, in s-SFTJ for the antiparallel alignment between the magnetization of the barrier and the magnetization of the reference layer, polarization inversion allows higher current densities, which results in larger torque amplitudes compared to the parallel configuration, see Figs. 4(a) and 4(c).

\section{Spin filter tunnel junctions with insulating spacers}

In Sec. III A, the bias dependence of partial spin filter tunnel junctions (NM/MI/FM) was studied. A dramatic enhancement of the spin-torque components compared to magnetic tunnel junctions was uncovered, as well as a domination of the fieldlike term compared to the damping torque $\left(T_{\perp}>T_{\|}\right)$. It was additionally found that the efficiency of $T_{\|}$remains in same order as in MTJs, whilst the efficiency of $T_{\perp}$ is dramatically enhanced. In Sec. III B, the angular dependence of symmetric structures of the form FM/MI/FM was studied, and we showed that depending on the relative orientation of the magnetizations in the barrier and free layer the system behaves like a MTJ or partial-SFTJ. These results may be put under debate since realistic structures require additional spacers to break the exchange interaction of the magnetic insulator and the ferromagnetic electrode; for this reason, we study here two architectures, a partial SFTJ of the form NM/MI/S/FM, and a symmetric junction of the form FM/S/MI/S/FM, where $\mathrm{S}$ stands for the insulating spacer defined by $N_{S}$ atomic layers. The barrier height of the spacer is set to $\epsilon_{S}^{0}=+7 \mathrm{eV}$ and $\Delta_{S}=0$, which guarantees large current densities without reaching the resonant regime. We also set $\epsilon_{B}^{0}=+9 \mathrm{eV}$ and $\Delta_{B}=+2 \mathrm{eV}$ and fix the other parameters as in the previous sections. It is found that insertion of insulating spacers does not qualitatively change our conclusions. In Fig. 5 main panels (insets), we display the bias dependence of the spin-torque efficiencies (components) for an MTJ with $N_{I}=3$ and p-SFTJs with $N_{\mathrm{MI}}=3$ and $N_{S}=0,1$, and 2 . Only the half-metallic case is given, but similar results are obtained in other band filling cases. Whereas in MTJs, the insertion of a spacer between the barrier and free layer modifies the quadratic profile of the fieldlike torque by inducing a linear term in V [see Eq. (2)], in p-SFTJs, spacers do not affect the linear profile reported in Fig. 2(b); moreover, because the torque is driven by the spin-dependent reflection, its amplitude exhibits a small decrease, which for $N_{S}=2$ remains still higher than MTJs, see inset in Fig. 5(b). In contrast, the in-plane torque being driven by the tunneling electrons that experience the lowest barrier height, this component dramatically decreases with the insertion of an insulating spacer [inset of Fig. 5(a)]. These results are confirmed 




FIG. 5. (Color online) MTJ and p-SFTJ efficiencies for the nonequilibrium (a) Slonczewski and (b) fieldlike spin-torque components as a function of bias. Three p-SFTJ structures of the form $\mathrm{NM} / \mathrm{MI} / \mathrm{S} / \mathrm{FM}$ are studied, where the thickness of MI is fixed to $N_{\mathrm{MI}}=3$ and of $\mathrm{S}$ to $N_{S}=0,1$, or 2 monolayers. The barrier thickness of the MTJ is set to $N_{I}=3$. Insets refer to the bias dependence of the nonequilibrium (top) Slonczewski and (bottom) fieldlike torque. In all cases, $\epsilon_{L(R)}^{0}=+5 \mathrm{eV}, \epsilon_{B}^{0}=+9 \mathrm{eV}, \theta_{R}=\pi / 2, \theta_{L(B)}=0$, and $t=-1 \mathrm{eV} . \epsilon_{S}^{0}=+7 \mathrm{eV}, \Delta_{R(B)}=+2 \mathrm{eV}$, and $\Delta_{L(S)}=0$ in p-SFTJs. $\Delta_{L(R)}=+2 \mathrm{eV}$ and $\Delta_{B}=0$ in MTJs.

by considering the torque efficiencies, displayed in main panels. Whereas the efficiency of the in-plane torque, $T_{\|} / j_{e}$, remains unaffected by the insertion of the spacer $(\sim-0.079)$, the efficiency of the perpendicular torque, $\left(T_{\perp}-T_{\perp}^{0}\right) / j_{e}$, increases; consequently, $T_{\perp} \gg T_{\|}$when insulating spacers are taken into consideration. Indeed, the dramatic enhancement of the spin-torque magnitudes in p-SFTJs is reduced in the presence of insulating spacers; however, when considering thin spacers the predicted spin torque is still higher than in MTJs.

We have extended this study to the case of a symmetric spin filter tunnel junction of the form FM/S/MI/S/FM with $N_{\mathrm{MI}}=3$ and $N_{S}=1$. The barrier heights are set to $\epsilon_{B}^{0}=+9 \mathrm{eV}$ and $\epsilon_{S}^{0}=+7 \mathrm{eV}$ for the magnetic insulator and the spacers, respectively. We found that the angular dependence of the torque becomes qualitatively similar to the case discussed in Sec. IIIB, where insulating spacers are neglected (not shown). In Fig. 6, the STT bias dependence of this symmetric junction in the low band filling regime $\left(\epsilon_{L(R)}^{0}=+3 \mathrm{eV}\right)$ is displayed for two configurations, $\theta_{B}=0$ and $\theta_{B}=3 \pi / 2$. The leads are set to $\theta_{L(R)}=\pi / 2(0)$, see top sketches in Fig. 6 . The former case $\left(\theta_{B}=0\right)$ defines a symmetric SFTJ with MTJ-like characteristics. Here, we compare our solutions with two types of MTJs with similar barrier thicknesses, $N_{I}=5$, and different barrier heights, $\epsilon_{B}^{0}=+7$ and $+9 \mathrm{eV}$. When the barrier height is closer to the Fermi level, the torque amplitudes are much larger; therefore, the MTJ with $\epsilon_{B}^{0}=$
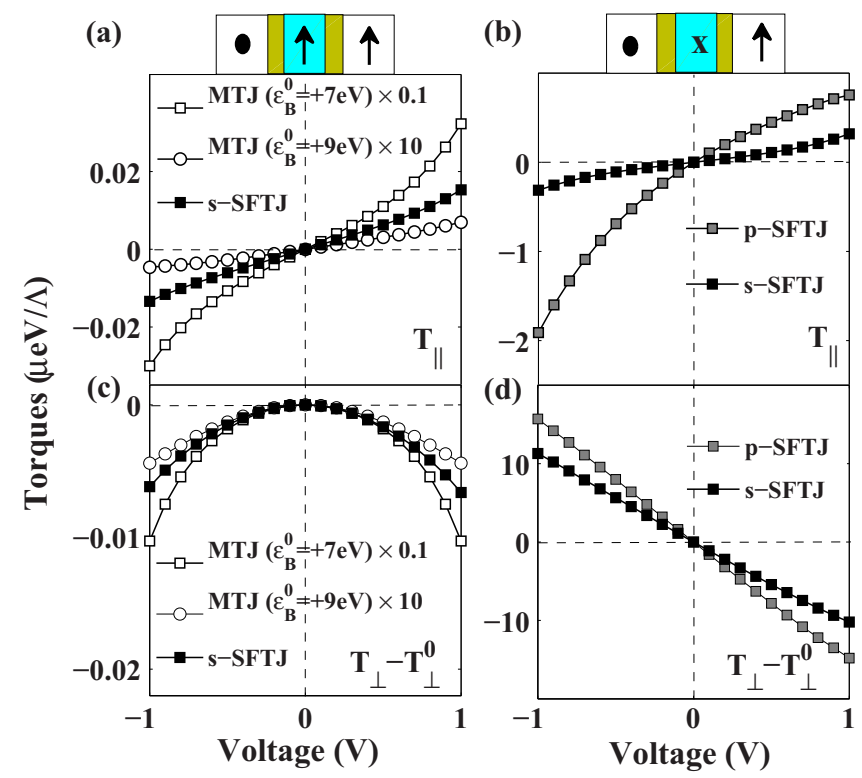

FIG. 6. (Color online) Bias dependence of the spin-torque components for $1 / 4$ band filling case $\left(\epsilon_{L(R)}^{0}=+3 \mathrm{eV}\right)$. Left (right) panels compare the s-SFTJ system of the form FM/S/MI/S/FM with a MTJ (p-SFTJ) structure of the form FM/I/FM (NM/MI/S/FM), where the thicknesses of I, MI, and S are fixed to $N_{I(\mathrm{MI})(S)}=5(3)(1)$. Top (bottom) panels refer to the nonequlibrium Slonczewski (fieldlike) component. The magnetization of the leads are kept perpendicular to each other, $\theta_{L(R)}=\pi / 2(0)$. Left (right) panels refer to the configuration where $\theta_{B}=0(3 \pi / 2)$. Two MTJ structures are displayed on left panels, $\epsilon_{B}^{0}=+7 \mathrm{eV}$ and $\epsilon_{B}^{0}=+9 \mathrm{eV}$. To fit in the graph, the former has been multiplied by 0.1 and the latter by 10 . Other parameters are similar to previous figures.

$+7 \mathrm{eV}\left(\epsilon_{B}^{0}=+9 \mathrm{eV}\right)$ shows much larger (lower) amplitudes than the symmetric SFTJ, as given in Figs. 6(a) and 6(c). If an MTJ of the form $\mathrm{FM} / \mathrm{S} / \mathrm{I} / \mathrm{S} / \mathrm{FM}$ is considered with similar parameters as the symmetric SFTJ, the latter will show torque amplitudes slightly enhanced due to the spin filtering imposed by the magnetic insulator (not show), in clear agreement with the ideal case given in Fig. 4(d) left panels. The latter case $\left(\theta_{B}=3 \pi / 2\right)$, which defines a symmetric SFTJ with p-SFTJ-like characteristics becomes more interesting. Here, the Slonczewski torque amplitude in the symmetric structure is lowered with respect to partial SFTJs of the form $\mathrm{NM} / \mathrm{MI} / \mathrm{S} / \mathrm{FM}$ with $N_{\mathrm{MI}}=3$ and $N_{S}=1$, in contrast to the ideal case given in Fig. 4(d) top right panel where both, symmetric and partial junctions showed similar amplitudes. This is due to the extra spacer added between the reference and barrier layers which contributes to an exponential decay of the electrons tunneling. Despite this decay, the magnitude is still larger than in MTJs with $N_{I}=3$, see Fig. 6(b) and inset in Fig. 2(a). Meanwhile, the fieldlike torque amplitude remains much larger than in MTJs and similar to partial SFTJs of the form NM/MI/S/FM because the contribution of electrons incident from the free layer (right to left) dominates; consequently, even if thick spacers are considered to decouple the reference and barrier layers, the torque magnitude shall not decrease significantly, see Fig. 6(d). 


\section{CONCLUSION}

To conclude, using a tight-binding model, we have theoretically studied the spin-transfer torque mechanism in junctions involving a magnetic insulating barrier. In the case of partial spin filter tunnel junction, composed of a magnetic tunnel barrier adjacent to a metallic ferromagnet, NM/MI/FM, we have shown a strong enhancement of the torque amplitudes compared to conventional MTJs. When an insulating spacer is added to decouple the exchange interaction between MI and FM, i.e., NM/MI/S/FM, we have shown that for thin spacers made of 1 or 2 monolayers, the torque amplitudes remain larger than conventional MTJs. The fieldlike torque strongly depends on the spin-dependent reflection at MI/FM interface. Meanwhile, the in-plane torque is driven by the tunneling electrons that experience the lowest barrier height. To exploit these important features and those of conventional MTJs, a hybrid device has been proposed, referred to as symmetric spin filter tunnel junction, and composed of two ferromagnetic electrodes separated by a magnetic tunneling barrier, FM/(S)/MI/(S)/FM. In such structure, we have shown that the STT components can be tuned from conventional MTJ-like to partial SFTJ-like behavior just by rotating the magnetization of the insulating layer. This offers the possibility of fabricating a device that is able to retrieve bias characteristics of both systems, partial SFTJ and conventional MTJ, which opens novel avenues in the future developments of current-induced-magnetization-reversal and -excitation devices.
[1] D. C. Ralph and M. D. Stiles, J. Magn. Magn. Mater. 320, 1190 (2008).

[2] J. C. Slonczewski, J. Magn. Magn. Mater. 159, L1 (1996); L. Berger, Phys. Rev. B 54, 9353 (1996).

[3] Yiming Huai, AAPPS Bull. 18, 6 (2008); S. I. Kiselev, J. C. Sankey, I. N. Krivorotov, N. C. Emley, R. J. Schoelkopf, R. A. Buhrman, and D. C. Ralph, Nature (London) 425, 380 (2003).

[4] M. Tsoi, A. G. M. Jansen, J. Bass, W.-C. Chiang, M. Seck, V. Tsoi, and P. Wyder, Phys. Rev. Lett. 80, 4281 (1998); 81, 493(E) (1998); E. B. Myers, D. C. Ralph, J. A. Katine, R. N. Louie, and R. A. Buhrman, Science 285, 867 (1999); J. A. Katine, F. J. Albert, R. A. Buhrman, E. B. Myers, and D. C. Ralph, Phys. Rev. Lett. 84, 3149 (2000).

[5] Yiming Huai, Frank Albert, Paul Nguyen, Mahendra Pakala, and Thierry Valet, Appl. Phys. Lett. 84, 3118 (2004); G. D. Fuchs, N. C. Emley, I. N. Krivorotov, P. M. Braganca, E. M. Ryan, S. I. Kiselev, J. C. Sankey, D. C. Ralph, R. A. Burman, and J. A. Katine, ibid. 85, 1205 (2004).

[6] J. Hayakawa, S. Ikeda, Y. M. Lee, R. Sasaki, T. Meguro, F. Matsukura, H. Takahashi, and H. Ohno, Jpn. J. Appl. Phys. Exp. Lett. 44, L1267 (2005); Z. Diao, D. Apalkov, M. Pakala, Y. Ding, A. Panchula, and Y. Huai, Appl. Phys. Lett. 87, 232502 (2005).

[7] W. H. Butler, X.-G. Zhang, T. C. Schulthess, and J. M. MacLaren, Phys. Rev. B 63, 054416 (2001); J. Mathon and A. Umerski, ibid. 63, 220403 (2001).

[8] M. Bowen, V. Cros, F. Petroff, A. Fert, C. Martnez Boubeta, J. L. Costa-Kramer, J. V. Anguita, A. Cebollada, F. Briones, J. M. de Teresa, L. Morelln, M. R. Ibarra, F. Guell, F. Peir, and A. Cornet, Appl. Phys. Lett. 79, 1655 (2001); S. Yuasa, T. Nagahama, A. Fukushima, Y. Suzuki, and K. Ando, Nat. Mater. 3, 868 (2004); S. S. P. Parkin, C. Kaiser, A. Panchula, P. M. Rice, B. Hughes, M. Samant, and S.-H. Yang, ibid. 3, 862 (2004); S. Yuasa and D. D. Djayaprawira, J. Phys. D: Appl. Phys. 40, R337 (2007).

[9] C. Heiliger and M. D. Stiles, Phys. Rev. Lett. 100, 186805 (2008); M. Wilczynski, J. Barnas, and R. Swirkowicz, Phys. Rev. B 77, 054434 (2008); J. Xiao, G. E. W. Bauer, and A. Brataas, ibid. 77, 224419 (2008); A. Manchon, N. Ryzhanova, N. Strelkov, A. Vedyayev, and B. Dieny, J. Phys. Condens. Matter 19, 165212 (2007).
[10] I. Theodonis, N. Kioussis, A. Kalitsov, M. Chshiev, and W. H. Butler, Phys. Rev. Lett. 97, 237205 (2006); I. Theodonis, N. Kioussis, A. Kalitsov, M. Chshiev, W. H. Butler, and A. Vedyayev, J. Appl. Phys. 99, 08G501 (2006); A. Kalitsov, M. Chshiev, I. Theodonis, N. Kioussis, and W. H. Butler, Phys. Rev. B 79, 174416 (2009); M. Chshiev, I. Theodonis, A. Kalitsov, N. Kioussis, and W. H. Butler, IEEE Trans. Mag. 44, 2543 (2008).

[11] P. M. Haney, C. Heiliger, and M. D. Stiles, Phys. Rev. B 79, 054405 (2009).

[12] S.-C. Oh, S.-Y. Park, A. Manchon, M. Chshiev, J.-H. Han, H.-W. Lee, J.-E. Lee, K.-T. Nam, Y. Jo, Y.-C. Kong, B. Dieny, and K.-J. Lee, Nat. Phys. 5, 898 (2009).

[13] Y.-H. Tang, N. Kioussis, A. Kalitsov, W. H. Butler, and R. Car, Phys. Rev. Lett. 103, 057206 (2009); Phys. Rev. B 81, 054437 (2010).

[14] A. Kalitsov, W. Silvestre, M. Chshiev, and J. P. Velev, Phys. Rev. B 88, 104430 (2013).

[15] A. Manchon, S. Zhang, and K.-J. Lee, Phys. Rev. B 82, 174420 (2010).

[16] Y.-H. Tang and N. Kioussis, Phys. Rev. B 85, 104413 (2012).

[17] W. Eerenstein, N. D. Mathur, and J. F. Scott, Nature (London) 442, 759 (2006); H. Bea, M. Gajek, M. Bibes, and A. Barthélémy, J. Phys. Condens. Matter 20, 434221 (2008).

[18] J. S. Moodera, X. Hao, G. A. Gibson, and R. Meservey, Phys. Rev. Lett. 61, 637 (1988).

[19] J. S. Moodera, J. Phys. Condens. Matter. 19, 165202 (2007).

[20] K. Uchida, J. Xiao, H. Adachi, J. Ohe, S. Takahashi, J. Ieda, T. Ota, Y. Kajiwara, H. Umezawa, H. Kawai, G. E. W. Bauer, S. Maekawa, and E. Saitoh, Nat. Mater. 9, 894 (2010); Y. Kajiwara, K. Harii, S. Takahashi, J. Ohe, K. Uchida, M. Mizuguchi, H. Umezawa, H. Kawai, K. Ando, K. Takanashi, S. Maekawa, and E. Saitoh, Nature (London) 464, 262 (2010).

[21] N. Jutong, I. Rungger, C. Schuster, U. Eckern, S. Sanvito, and U. Schwingenschlögl, Phys. Rev. B 86, 205310 (2012).

[22] D. C. Worledge and T. H. Geballe, J. Appl. Phys. 88, 5277 (2000); Z.-W. Xie and B.-Z. Li, ibid. 93, 9111 (2003); M. Wilczynski, J. Magn. Magn. Mater. 325, 94 (2013).

[23] A. Saffarzadeh, J. Magn. Magn. Mater. 269, 327 (2004); M G. Chapline and S. X. Wang, J. Appl. Phys. 100, 123909 (2006). 
[24] D. Jin, Y. Ren, Z.-Z. Li, M.-W. Xiao, G. Jin, and A. Hu, Phys. Rev. B 73, 012414 (2006).

[25] P. LeClair, J. K. Ha, H. J. M. Swagten, J. T. Kohlhepp, C. H. van de Vin, and W. J. M. de Jonge, Appl. Phys. Lett. 80, 625 (2002); G.-X. Miao, M. Muller, and J. S. Moodera, Phys. Rev. Lett. 102, 076601 (2009).

[26] J. S. Moodera, R. Meservey, and X. Hao, Phys. Rev. Lett. 70, 853 (1993).

[27] T. S. Santos and J. S. Moodera, Phys. Rev. B 69, 241203(R) (2004).

[28] M. G. Chapline and S. X. Wang, Phys. Rev. B 74, 014418 (2006); A. V. Ramos, M. J. Guittet, J. B. Moussy, R. Mattana, C. Deranlot, F. Petroff, and C. Gatel, Appl. Phys. Lett. 91, 122107 (2007).

[29] U. Luders, M. Bibes, K. Bouzehouane, E. Jacquet, J.-P. Contour, S. Fusil, J.-F. Bobo, J. Fontcuberta, A. Barthélémy, and A. Fert, Appl. Phys. Lett. 88, 082505 (2006); U. Luders, M. Bibes, S. Fusil, K. Bouzehouane, E. Jacquet, C. B. Sommers, J.-P. Contour, J.-F. Bobo, A. Barthélémy, A. Fert, and P. M. Levy, Phys. Rev. B 76, 134412 (2007).

[30] B. B. Nelson-Cheeseman, R. V. Chopdekar, L. M. B. Alldredge, J. S. Bettinger, E. Arenholz, and Y. Suzuki, Phys. Rev. B 76, 220410(R) (2007).
[31] M. Gajek, M. Bibes, A. Barthélémy, K. Bouzehouane, S. Fusil, M. Varela, J. Fontcuberta, and A. Fert, Phys. Rev. B 72, 020406(R) (2005).

[32] R. V. Chopdekar, B. B. Nelson-Cheeseman, M. Liberati, E. Arenholz, and Y. Suzuki, Phys. Rev. B 83, 224426 (2011).

[33] J.-I. Inoue, J. Appl. Phys. 111, 07 C902 (2012); Phys. Rev. B 84, 180402(R) (2011).

[34] L. V. Keldysh, Sov. Phys. JETP 20, 4 (1965); C. Caroli, R. Combescot, P. Nozieres, and D. Saint-James, J. Phys. C 4, 916 (1971).

[35] H. Kubota, A. Fukushima, K. Yakushiji, T. Nagahama, S. Yuasa, K. Ando, H. Maehara, Y. Nagamine, K. Tsunekawa, D. D. Djayaprawira, N. Watanabe, and Y. Suzuki, Nat. Phys. 4, 37 (2008); J. C. Sankey, Y.-T. Cui, J. Z. Sun, J. C. Slonczewski, R. A. Buhrman, and D. C. Ralph, ibid. 4, 67 (2008); C. Wang, Y.-T. Cui, J. Z. Sun, J. A. Katine, R. A. Buhrman, and D. C. Ralph, Phys. Rev. B 79, 224416 (2009).

[36] W. H. Butler, Sci. Technol. Adv. Mater. 9, 014106 (2008).

[37] T. Nagahama, T. S. Santos, and J. S. Moodera, Phys. Rev. Lett. 99, 016602 (2007); G. X. Miao and J. S. Moodera, J. Appl. Phys. 106, 023911 (2009).

[38] J. C. Slonczewski, Phys. Rev. B 39, 6995 (1989). 